\title{
Efficacy of second-generation antipsychotics in patients at ultra-high risk and those with first-episode or multi-episode schizophrenia
}

This article was published in the following Dove Press journal:

Neuropsychiatric Disease and Treatment

18 June 2013

Number of times this article has been viewed

\author{
Kenji Washida ${ }^{1,2}$ \\ Toshihiko Takeda ${ }^{2}$ \\ Toshiaki Habara ${ }^{2}$ \\ Soichiro Sato ${ }^{2}$ \\ Takuro Oka ${ }^{2}$ \\ Masuo Tanaka ${ }^{2}$ \\ Yusaku Yoshimura ${ }^{2}$ \\ Shozo Aoki' \\ 'Department of Psychiatry, Kawasaki \\ Medical Graduate School, Kurashiki, \\ Japan; ${ }^{2}$ Zikei Hospital, Okayama, Japan
}

Correspondence: Kenji Washida Zikei Hospital, 100-2 Urayasu-honmachi, Minami-ku, Okayama, 702-8508, Japan

$\mathrm{Tel}+8 \mid 86262$ II 9 I

Fax +8I 862624448

Email nodokanahiyori@zikei.or.jp
Aim: The aim of this study was to examine the speed of response, doses, and safety of treatment with second-generation antipsychotics (SGAs) in patients at ultra-high risk (UHR) compared to those with schizophrenia.

Methods: A 12-week open-label, prospective study of SGAs was performed in UHR patients and those with first-episode schizophrenia (FES) and multi-episode schizophrenia (MES). The subjects were 14-30 years old and were recruited at Zikei Hospital, Okayama, Japan from December 1, 2006 to December 1, 2011. Treatment was carried out in a natural setting in an open-label format, but clinical evaluation was performed blind. The clinical rating scales include the Global Assessment of Functioning (GAF), the Positive and Negative Syndrome Scale (PANSS), and the Clinical Global Impression-Severity scale (CGI-S).

Results: UHR $(n=17)$, FES $(n=23)$, and MES $(n=21)$ patients all showed significant improvements on the GAF, PANSS, and CGI-S. However, the UHR patients showed significantly greater improvement on the GAF at weeks 4, 8, and 12 compared to the other groups, and a significantly lower modal dose of SGAs (chlorpromazine equivalent: 183 [201.1] mg/day, mean [SD]) was needed for improvement in the UHR group. Each group was also prescribed anticholinergic agents during the study period and the UHR group had significantly fewer extrapyramidal symptoms (only 6\%) compared with the FES group.

Conclusion: Our findings suggest that UHR patients have a better response to SGAs compared to patients with schizophrenia, and that these drugs can be given safely by minimizing the dosage of SGAs and using anticholinergic agents.

Keywords: ultra-high risk, second-generation antipsychotics, schizophrenia

\section{Introduction}

Several studies have investigated the efficacy of early interventions for prevention of the transition to psychosis, including schizophrenia. Patients with possible onset are prospectively identified as being in an at-risk mental state (ARMS) $)^{1}$ and early interventions have been carried out for those identified as ultra-high risk (UHR) for development of schizophrenia or other psychotic disorders. ${ }^{2,3}$ Early intervention is supported by results for the duration of untreated psychosis in schizophrenia. Thus, patients have a better prognosis with a shorter period from the onset of illness to intervention, ${ }^{4,5}$ and early detection before disease progression may have a good influence on the prognosis.

UHR is diagnosed using the Comprehensive Assessment of At-Risk Mental States (CAARMS) or the Structured Interview for Prodromal Syndromes (SIPS). ${ }^{1,6}$ Yearly rates of $13 \%$ to $50 \%$ for progression to psychosis have been reported. ${ }^{7}$ Interventions for 
UHR patients mainly involve pharmacotherapy and cognitive behavior therapy (CBT). ${ }^{8}$ Second-generation antipsychotics (SGAs) and antidepressants are prescribed for UHR patients, ${ }^{9,10}$ but their use is restricted as first-line pharmacotherapy. ${ }^{8}$ Antipsychotics are useful for urgent improvement in a crisis, such as threatened self-mutilation and suicide, and SGAs are effective for preventing or delaying progression to psychosis. ${ }^{9,11-14}$

Ethical considerations regarding false positive identification of suspected prodromal patients and adverse reactions related to pharmacotherapy are of concern. ${ }^{9,11,15}$ It is difficult to distinguish a false positive case at the start of drug treatment using current diagnostic tests, and this prevents a consensus on the appropriate medication for the UHR patient population, in which false positive cases are intermingled. Therefore, the aim of this study was to examine the speed of response, doses, and safety of treatment with SGAs in patients at UHR compared to those with schizophrenia.

\section{Methods}

\section{Subjects}

The subjects were inpatients and outpatients aged 14-30 years old who visited Zikei Hospital, Okayama, Japan from December 1, 2006 to December 1, 2011. The UHR group was diagnosed using the CAARMS, and the first-episode schizophrenia (FES) and multi-episode schizophrenia (MES) groups were diagnosed based on the International Classification of Diseases (10th revision). ${ }^{16}$ Exclusion criteria were: cases in which no consent was obtained; pregnancy; IQ under 70; neurological disorder and drug dependence; mood disorders; pervasive developmental disorders; conduct disorders; and personality disorders. Patients with UHR and FES had not been prescribed antipsychotics for more than 2 weeks before the start of the study. For matching with the other two groups, patients with MES with a Global Assessment of Functioning (GAF) score under 70 before relapse and with treatment-resistant disease in the last episode were excluded from the study. All subjects in the study were prescribed psychotropic drugs.

\section{Treatment}

Treatment was performed prospectively in a 12-week openlabel study. A flexible dosing strategy was applied using a clinically relevant algorithm. The UHR group was prescribed SGAs, antidepressants, or benzodiazepines as the main agent, and the FES and MES groups were prescribed SGAs as the main agent. Augmentation and concomitant medications were permitted based on clinical judgment. Other antipsychotics used for augmentation were used less than the main agent and were decreased or stopped when possible during the study. Anticholinergic agents were reduced to minimum dosages during the study period, but preventive prescription was allowed. Supportive psychotherapy and occupational therapy were also performed as standard medical treatment during the study.

\section{Clinical assessment}

Clinical evaluation was performed by raters other than physicians at baseline and after 4, 8, and 12 weeks of treatment. If treatment was ended before 12 weeks, an evaluation was performed at the end point. The clinical rating scales used included the Positive and Negative Syndrome Scale (PANSS), ${ }^{17}$ GAF, ${ }^{18}$ Clinical Global Impression-Severity scale (CGI-S), ${ }^{19}$ Simpson-Angus Scale (SAS), ${ }^{20}$ Barnes Akathisia Rating Scale (BARS), ${ }^{21}$ and Abnormal Involuntary Movement Scale (AIMS). ${ }^{22}$ Adverse events were documented and switching of SGAs was evaluated based on reasons of efficacy and tolerability.

\section{Ethics}

The investigation was carried out in accordance with the latest version of the Declaration of Helsinki. The study design was approved by the medical ethics committee of Zikei Hospital. Informed consent was obtained from each patient. For patients who were minors, the consent of one or more guardians was also obtained.

\section{Statistical analysis}

Differences in ratios among the three groups were evaluated by $\chi^{2}$ and Fisher's exact test. Differences in data over time within groups were evaluated by Friedman test and differences among the three groups at each time point were evaluated by KruskalWallis test. If significant differences were found by KruskalWallis test, comparison among the three groups at each time point was performed by Steel-Dwass test. Differences in the doses of antipsychotic drugs at individual time points within groups were evaluated by Mann-Whitney $U$ test. $P<0.05$ was considered to be significant in all analyses.

\section{Results}

\section{Baseline characteristics}

Demographic details of the three groups are shown in Table 1. A total of 72 patients were registered at Zikei Hospital, but only 61 (UHR $=17, \mathrm{FES}=23$, MES $=21$ ) were included as subjects because only cases treated with an antipsychotic drug as the main agent were eligible for this study. The numbers of males and females were almost equal, and 48 subjects were inpatients. There were no significant differences in gender 
Table I Demographic details of the UHR, FES, and MES groups

\begin{tabular}{|c|c|c|c|c|c|}
\hline & UHR & FES & MES & $P$ & $\begin{array}{l}\text { Steel-Dwass } \\
\text { test }(P<0.05)\end{array}$ \\
\hline $\mathrm{N}$ & 17 & 23 & 21 & & \\
\hline $\operatorname{Sex}(M / F)$ & $5 / 12$ & $14 / 9$ & $11 / 10$ & 0.13 & \\
\hline Outpatients/inpatients & $8 / 9$ & $2 / 21$ & $3 / 18$ & & \\
\hline Age, y & $23.7(4.4)$ & $23.3(4.6)$ & $26.3(3.9)$ & $0.041 *$ & FES $<$ MES \\
\hline Age at onset & $23.7(4.4)$ & $23.3(4.6)$ & $22.2(4.2)$ & $0.6^{*}$ & \\
\hline GAF & $29.7(I 3.5)$ & $24.3(12.0)$ & $35.7(16.6)$ & $0.036 *$ & FES $<$ MES \\
\hline PANSS & $72.0(20.0)$ & 99.1 (24.9) & $81.9(23.7)$ & $0.002 *$ & UHR $<$ FES \\
\hline Positive symptoms & $17.4(7.2)$ & $26.4(8.6)$ & $24(9.4)$ & $0.001 *$ & UHR $<$ FES MES \\
\hline Negative symptoms & $12.2(5.2)$ & $20.4(11.0)$ & $16.1(8.1)$ & $0.037^{*}$ & UHR $<$ FES \\
\hline General symptoms & $42.4(12.5)$ & $52.2(13.0)$ & $4 I .7(12.6)$ & $0.01 *$ & UHR MES $<$ FES \\
\hline CGI-S & $4.5(1.1)$ & $5.5(0.9)$ & $5.0(1.0)$ & $0.012 *$ & UHR $<$ FES \\
\hline
\end{tabular}

Notes: Data are presented as mean (standard deviation), except where indicated otherwise. Differences in three subgroups with $P$-values calculated by $\chi^{2}$ test (sex). *Kruskal-Wallis test.

Abbreviations: CGI-S, Clinical Global Inventory-Severity; FES, first-episode schizophrenia; GAF, Global Assessment of Functioning; MES, multi-episode schizophrenia; PANSS, Positive and Negative Syndrome Scale; UHR, ultra-high risk; y, years.

among the three groups. The MES group was older than the FES group based on age at the time of entry into the study, but there were no significant differences in age at onset among the three groups. The GAF score was significantly lower in the FES group compared to the MES group, but did not differ significantly between the UHR and FES groups. The PANSS total score was significantly higher in the FES group compared with the UHR group. The positive symptoms score in the UHR group was significantly lower than those in the two schizophrenia groups, while the negative symptoms score in the UHR group was significantly lower than that in the FES group. The general symptoms scores in the UHR and MES groups were significantly lower than that in the FES group. CGI-S scores were significantly higher in the FES group compared with the UHR group.

\section{GAF}

GAF scores improved significantly in the UHR, FES, and MES groups after 12 weeks (Table 2). There was no

Table 2 Results for the GAF, PANSS, CGI-S, and dose of SGAs

\begin{tabular}{|c|c|c|c|c|c|}
\hline Weeks & UHR & FES & MES & $P *$ & $\begin{array}{l}\text { Steel-Dwass } \\
\text { test }(\boldsymbol{P}<0.05)\end{array}$ \\
\hline \multicolumn{6}{|l|}{ GAF } \\
\hline Ow & $29.7(13.5)$ & $24.3(12.0)$ & $35.7(16.6)$ & 0.036 & FES $<$ MES \\
\hline $4 w$ & 64.1 (12.9) & 43.5 (19.6) & 47.1 (12.4) & 0.0007 & UHR > FES MES \\
\hline $8 w$ & $67.2(11.8)$ & $53.8(13.2)$ & $51.6(9.9)$ & 0.0011 & UHR $>$ FES MES \\
\hline $12 w$ & $70.8(9.7)$ & 58.1 (15.7) & $56.1(9.2)$ & 0.0067 & UHR $>$ FES MES \\
\hline \multicolumn{6}{|l|}{ PANSS } \\
\hline $0 w$ & $72.0(20.0)$ & 99.1 (24.9) & $81.9(23.7)$ & 0.002 & UHR $<$ FES \\
\hline $4 w$ & $50.1(13.8)$ & $71.9(28.3)$ & $69.9(18.6)$ & 0.0062 & UHR $<$ FES MES \\
\hline $8 w$ & $45.3(12.0)$ & $60.1(19.5)$ & $62.7(16.1)$ & 0.0029 & UHR $<$ FES MES \\
\hline $12 w$ & $41.3(7.7)$ & $53.2(19.6)$ & $58(18.6)$ & 0.0087 & UHR $<$ MES \\
\hline \multicolumn{6}{|l|}{ CGI-S } \\
\hline $0 w$ & $4.5(1.1)$ & $5.5(0.9)$ & $5.0(1.0)$ & 0.0124 & $\mathrm{UHR}<\mathrm{FES}$ \\
\hline $4 w$ & $2.8(0.6)$ & $4.2(I .5)$ & $4.1(0.8)$ & $<0.0001$ & UHR $<$ FES MES \\
\hline $8 w$ & $2.5(0.7)$ & $3.7(1.1)$ & $3.6(0.7)$ & 0.0002 & UHR $<$ FES MES \\
\hline $12 w$ & $2.3(0.8)$ & $3.1(1.2)$ & $3.3(1.1)$ & 0.0237 & UHR $<$ MES \\
\hline \multicolumn{6}{|c|}{ Dose of SGAs } \\
\hline $0 w$ & |9|.5 (|44.5) & $297.8(169.9)$ & $436.6(176.5)$ & & \\
\hline $4 w$ & I 68.3 (167.9) & $455.6(219.6)^{\dagger}$ & $568.4(244.4)$ & & \\
\hline $8 w$ & $177.0(|8| .2)$ & $406.0(177.9)^{\dagger}$ & $549.4(264.3)$ & & \\
\hline $12 w$ & | 40.8 (10|.5) & $346.2(190.5)$ & $534.7(254.3)$ & & \\
\hline
\end{tabular}

Notes: Data are presented as mean (standard deviation), except where indicated otherwise. Significant improvements occurred between each group over 12 weeks (Friedman test). GAF: UHR $P<0.0$ I; FES $P<0.0$ I; MES $P<0.01$. PANSS: UHR $P<0.0$ I; FES $P<0.01$; MES: $P<0.01$. CGI-S: UHR $P<0.01$; FES $P<0.0$ I; MES $P<0.0$ I. *Kruskal-Wallis test. There were significant differences between 0 week and 4 or 8 weeks in the FES group. ${ }^{\dagger}$ Mann-Whitney test, $P<0.02$.

Abbreviations: CGI-S, Clinical Global Inventory-Severity; FES, first-episode schizophrenia; GAF, Global Assessment of Functioning; MES, multi-episode schizophrenia; PANSS, Positive and Negative Syndrome Scale; SGAs, second-generation antipsychotics; UHR, ultra-high risk. 
significant difference in the baseline GAF score between the UHR group and the two schizophrenia groups, but the GAF scores at 4, 8, and 12 weeks were higher in the UHR group. Therefore, UHR patients showed significantly faster and greater improvement in GAF compared to those with FES and MES (Table 2 and Figure 1). UHR patients showed rapid improvement from an average score of 29.7 at baseline to 64.1 at 4 weeks after initiation of therapy. In contrast, the GAF score in the FES group only improved from 24.3 at baseline to 43.5 at 4 weeks, at which time there was already a significant difference between the UHR and FES groups. Similarly, the GAF score in the MES group only improved from 35.7 at baseline to 47.1 at 4 weeks, again with a significant difference between the UHR and MES groups at 4 weeks.

\section{PANSS}

PANSS scores showed significant improvements in all three groups (Table 2). There were significant differences in the PANSS score until 8 weeks between the UHR and FES groups, but this difference was lost at 12 weeks. This result does not indicate that the UHR group showed less improvement than the FES group in the later part of the study; rather, the UHR group had already shown a major improvement on PANSS by an average of 50 points after 4 weeks and further improvement was unlikely. The early improvement of the UHR group was also shown by the significant improvement in PANSS scores after 4 weeks for this group compared to the MES group, given that the PANSS scores in these groups did not differ significantly at baseline.

\section{CGI-S}

The tendency for early improvement in UHR patients was also seen on the CGI-S (Table 2). In the UHR group, all scores were $<3$ points after 4 weeks, whereas CGI-S scores in the other two groups were $>3$ points, although these scores decreased over 12 weeks. Thus, UHR patients clearly had significantly better early improvement compared with patients with schizophrenia, based on the results for the GAF, PANSS, and CGI-S.

\section{Doses of SGAs}

There was no significant difference in the doses of SGAs given as chlorpromazine equivalents in the UHR group between the start and other points (Table 2). In contrast, the doses were significantly higher at weeks 4 and 8 in the FES group, compared to those at the start of the study. There was no significant difference in the doses between the start and other points in the MES group.

The means of the maximum doses of SGAs were 274.3 (239.9) (standard deviation [SD]) $\mathrm{mg}$ in the UHR group, 527.8 (240) $\mathrm{mg}$ in the FES group, and 745.3 (287.8) $\mathrm{mg}$ in the MES group, with significant differences among the three groups $(P<0.01$ Kruskal-Wallis test, $P<0.05$ Steel-Dwass test). The maximum doses in the UHR group

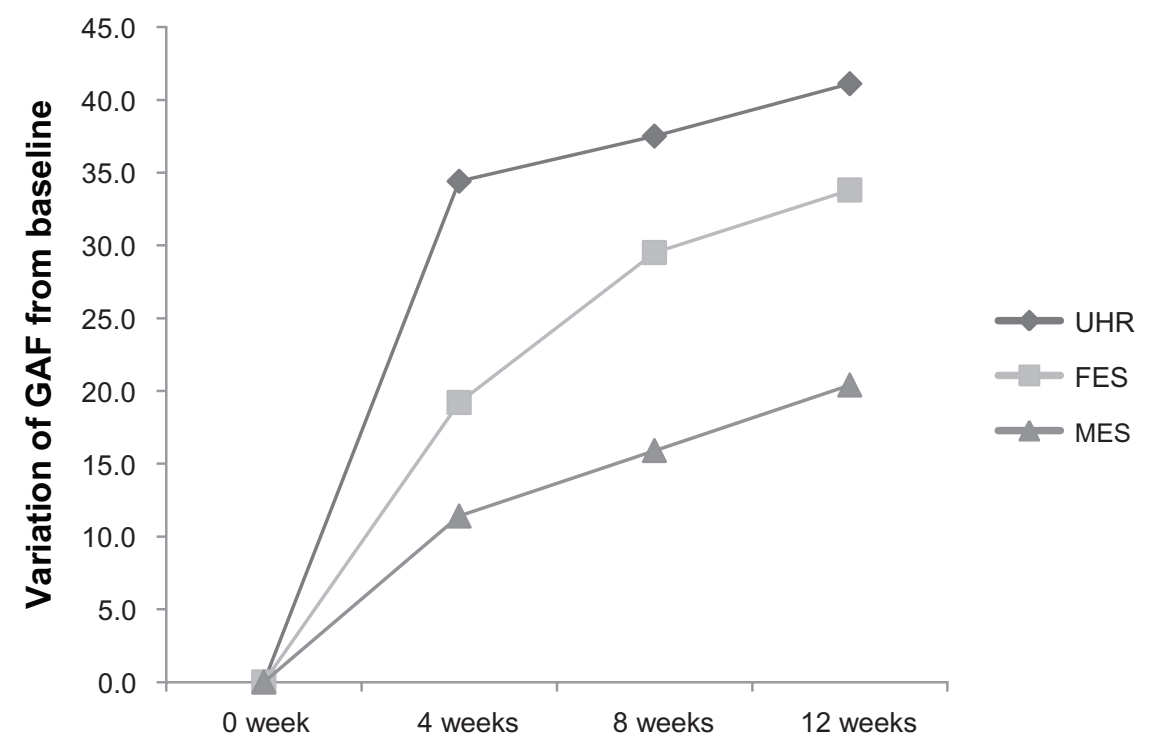

Figure I Improvement of GAF scores from baseline.

Notes: There were significant differences within all three groups: UHR $P<0.01$, FES $P<0.01$, MES $P<0.01$ (Friedman test). There were also significant differences at 4, 8, and I 2 weeks: GAF weeks 4, $8, I 2 P<0.0$ I (Kruskal-Wallis test). UHR 4 weeks $>$ FES 4 weeks MES 4 weeks; UHR 8 weeks FES 8 weeks $>$ MES 8 weeks; UHR I 2 weeks $>$ MES 12 weeks (Steel-Dwass test, $P<0.05$ ).

Abbreviations: FES, first-episode schizophrenia; GAF, Global Assessment of Functioning; MES, multi-episode schizophrenia; UHR, ultra-high risk. 
were significantly lower than those in the FES and MES groups, and the doses in the MES group were significantly the highest among the three groups.

The means of modal doses of SGAs were 183 (201.1) $\mathrm{mg}$ in the UHR group, 382.3 (191.9) $\mathrm{mg}$ in the FES group, and 574 (289.6) $\mathrm{mg}$ in the MES group (Figure 2), again with significant differences among the three groups. The modal doses in the UHR group required for improvement was significantly lower than that in the other two groups, while the MES group received significantly the highest doses among three groups.

\section{Switching of SGAs and adverse events}

Switching of SGAs was performed at a rate of almost $50 \%$ in all patients (Table 3 ). The rates for switching due to poor efficacy were $44 \%, 70 \%$, and $70 \%$ in the UHR, FES, and MES groups, respectively, and the rates for switching due to adverse events were $67 \%, 50 \%$, and $70 \%$ in the same respective groups. Each group was prescribed anticholinergic agents and the frequencies of extrapyramidal symptoms (EPSs) were $6 \%, 65 \%$, and $29 \%$ in the UHR, FES, and MES groups, respectively, over the whole study period. At the end of the study, these rates were $6 \%, 39 \%$, and $10 \%$, respectively. The FES group had a significantly higher frequency of EPSs compared with the other two groups. Adverse events other than EPSs included somnolence and fatigue in all three groups.

The type and number of SGAs prescribed to the three groups are shown in Table 4. During the study period, aripiprazole, olanzapine and risperidone were mainly prescribed as SGAs.

\section{Discussion}

We examined the speed of response, doses, and safety of treatment with SGAs in patients at UHR compared to those with schizophrenia. On the GAF, UHR patients showed significantly faster and greater improvement compared to those with FES and MES. In the CAARMS, UHR patients are defined as those with mild symptoms of schizophrenia. Therefore, the PANSS score in the UHR group had a tendency to be lower than those in the FES and MES groups. Although PANSS scores showed significant improvements in all three groups, those did not clarify about the speed of response to treatment in this study. GAF scores do not necessarily correlate with symptoms and give a low value in crisis patients that are acting out through self-mutilation and threatened suicide, or those with social withdrawal. Treatment of UHR patients is often performed in outpatient settings, but there were more inpatients than outpatients in this study, indicating that many cases were in a crisis. This study suggests that the GAF is a very sensitive rating scale for mild symptoms such as those in UHR patients. McGlashan et al have also suggested that PANSS is not sensitive enough to show improvement of illness in UHR cases. ${ }^{9}$

The CGI-S is a rating scale that can be used after evaluation with GAF and PANSS. The CGI-S results showed that UHR patients had already reached a good level after 4 weeks, with greater improvement at this time

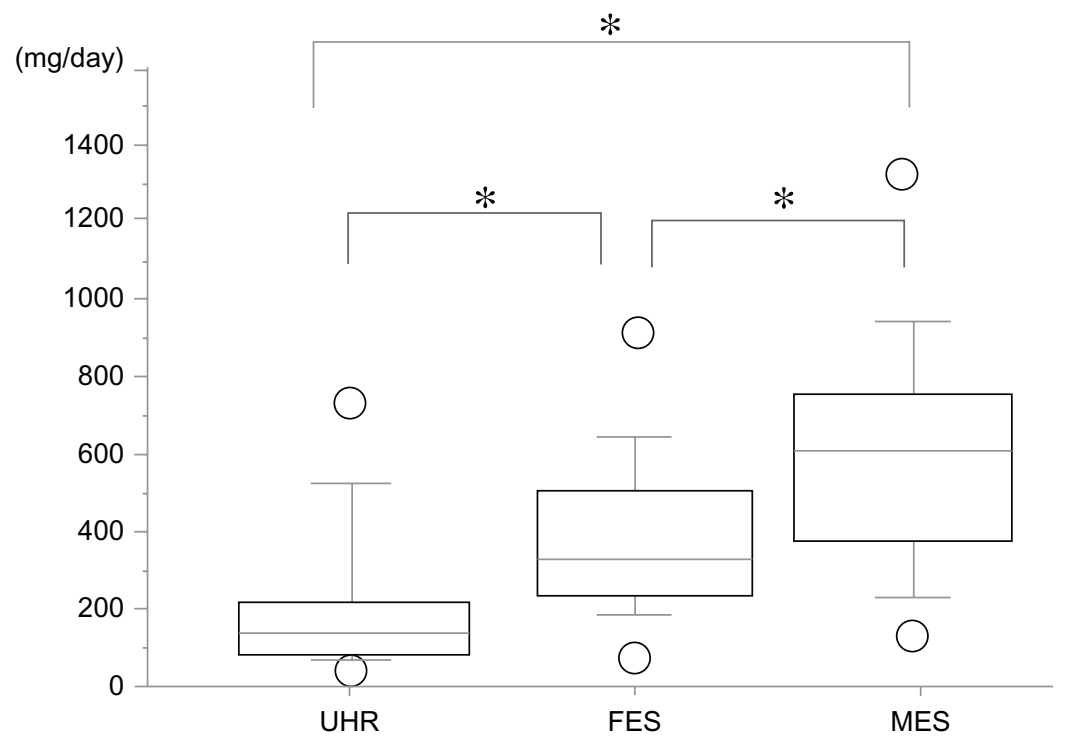

Figure 2 Modal doses of SGAs shown as chlorpromazine equivalents.

Notes: Mean (standard deviation) dose: UHR I83 (20I.I) mg/day, FES 382.3 (I9l.9) mg/day, MES 574 (289.6) mg/day, $P<0.01$ (Kruskal-Wallis test). *P $<0.05$ (Steel-Dwass test). Abbreviations: FES, first-episode schizophrenia; MES, multi-episode schizophrenia; SGAs, second-generation antipsychotics; UHR, ultra-high risk. 
Table 3 Switching of SGAs and adverse events

\begin{tabular}{|c|c|c|c|}
\hline & UHR & FES & MES \\
\hline The rates of switching & $53 \%$ & $48 \%$ & $48 \%$ \\
\hline \multicolumn{4}{|l|}{ The reason of switching } \\
\hline Poor efficacy & $44 \%$ & $70 \%$ & $70 \%$ \\
\hline Adverse events & $67 \%$ & $50 \%$ & $70 \%$ \\
\hline \multicolumn{4}{|l|}{ Anticholinergic agent } \\
\hline The whole study period & $24 \%$ & $43 \%$ & $29 \%$ \\
\hline The end of the study & $6 \%$ & $22 \%$ & $24 \%$ \\
\hline \multicolumn{4}{|l|}{ Extrapyramidal symptom (EPS) } \\
\hline The whole study period* & $6 \%$ & $65 \%$ & $29 \%$ \\
\hline The end of the study** & $6 \%$ & $39 \%$ & $10 \%$ \\
\hline \multicolumn{4}{|l|}{ Adverse events other than EPS } \\
\hline Somnolence and fatigue & $42 \%$ & $52 \%$ & $48 \%$ \\
\hline Constipation & $12 \%$ & $22 \%$ & $14 \%$ \\
\hline Dry mouth & $0 \%$ & $13 \%$ & $19 \%$ \\
\hline Increased appetite & $0 \%$ & $17 \%$ & $5 \%$ \\
\hline Menoxenia & $6 \%$ & $4 \%$ & $5 \%$ \\
\hline
\end{tabular}

Notes: FES $>$ UHR MES $\left(* P<0.01 \chi^{2}\right.$ for independence test, $P<0.017$ Fisher's exact test). FES $>$ UHR MES (**P $<0.012 \chi^{2}$ for independence test, $P<0.026$ for Fisher's exact test).

Abbreviations: EPSs, extrapyramidal symptoms; FES, first-episode schizophrenia; MES, multi-episode schizophrenia; SGAs, second-generation antipsychotics; UHR, ultra-high risk.

point compared with the other two groups. In the UHR group, ceiling effects for improvement of symptoms and functions were observed at week 4 . In contrast, patients with schizophrenia tended to show more gradual improvement over the duration of the study. A comparative study of the treatment response of patients with FES and recurrent schizophrenia has been reported, ${ }^{23,25-28}$ but there has been no previous comparison of UHR and schizophrenia patients. Thus, this is the first study to show faster improvement of functions and global impressions in UHR patients compared to those with FES and MES after treatment with SGAs, based on the GAF and CGI-S.

All the UHR patients were treated with SGAs as the main agent in this study. The doses of SGAs in the FES and MES groups were within the limits of the recommended doses for acute phase or recurrent acute-phase schizophrenia, ${ }^{24}$

Table 4 The type and number of SGAs prescribed to the UHR, FES, and MES groups

\begin{tabular}{lllll}
\hline & UHR & FES & MES & Total \\
\hline Aripiprazole & 9 & 9 & 8 & 26 \\
Blonanserin & 1 & 1 & 3 & 5 \\
Clozapine & 0 & 0 & 0 & 0 \\
Olanzapine & 5 & 13 & 8 & 26 \\
Paliperidone & 1 & 0 & 5 & 6 \\
Perospirone & 1 & 1 & 0 & 2 \\
Quetiapine & 2 & 5 & 9 & 16 \\
Risperidone & 6 & 8 & 8 & 22 \\
\hline
\end{tabular}

Abbreviations: FES, first-episode schizophrenia; MES, multi-episode schizophrenia; SGAs, second-generation antipsychotics; UHR, ultra-high risk. whereas the UHR group was treated at a dose close to the minimum recommended dose. Clinical studies on antipsychotics for UHR or ARMS patients ${ }^{9,1-14}$ have used doses (chlorpromazine equivalents) of approximately 100-375 mg/day. In the current study, the modal dose of SGAs prescribed in the UHR group was 183 (201.1) $\mathrm{mg}$ /day and the results were similar to those found in previous studies. These results show that UHR patients in various crises can be treated effectively with SGAs at a dose close to the minimum recommended for FES. ${ }^{30}$ Significant differences in the modal dose were also found among the groups, similarly to the maximum dose. Thus, the results suggest that UHR patients have a faster response to lower doses of SGAs compared to patients with schizophrenia.

Switching of SGAs was performed at a rate of almost $50 \%$ in all patients. In the UHR group, the rate of switching for adverse events was $67 \%$, but that for poor response was only $44 \%$. This reflects the good response of UHR patients to SGAs, and thus switching was based more on adverse events. In contrast, both a poor response and adverse events led to switching in the FES and MES groups. However, serious adverse events did not occur in any of the groups over 12 weeks.

Anticholinergic agents were permitted in the study, including preventive prescription before appearance of EPSs. Anticholinergic agents were prescribed at rates of $24 \%, 43 \%$, and $29 \%$ over the study period in the UHR, FES, and MES groups, respectively, but these rates had fallen to $6 \%, 22 \%$, and $24 \%$, respectively, by the end of the study. EPSs occurred at a rate of only $6 \%$ (one patient) in the UHR group, but at $65 \%$ and $29 \%$ in the FES and MES groups, respectively, over the whole study. In the FES and MES groups, these rates had fallen to $39 \%$ and $10 \%$, respectively, by the end of the study. Thus, the UHR group showed the most improvement of functions and global impressions and the lowest incidence of EPSs, and our findings suggest that SGAs can be administered safely in UHR patients by proper choice of the dosage and use of anticholinergic agents.

EPSs occurred in one subject (6\%) in the UHR group during the 12-week study period, although there were no serious side effects. Therefore, if UHR patients are prescribed SGAs at higher doses than necessary to improve symptoms, safety may be compromised and adverse events may reach levels observed in the FES group. Since sufficient doses of SGAs were already being prescribed upon commencement of the study in the UHR group, no large-scale titration of the dose of SGAs was required until completion of the study (Table 2). Pharmacotherapy in UHR may be possible with 
doses of SGAs lower than those shown in Figure 2 and a further detailed titration may be necessary in a shorter period of time. Moreover, SGAs caused less EPSs compared with first-generation antipsychotics (FGAs), and FGAs may increase the incidence of EPSs in UHR patients. There was a required increase in the doses of SGAs in the FES group because the symptoms at baseline were serious and there was insufficient improvement at weeks 4 and 8.

Age at baseline in the MES group tended to be higher than that in the FES group, although there was no significant difference in age of onset among the three groups. The slightly higher age in the MES group may cause the slow improvement of functions and global impressions.

Adverse events other than EPSs were mainly somnolence and fatigue in the three groups. All SGAs have some inhibitory effects on receptors related to sedation, such as the histamine or $\alpha 1$ receptor. A meta-analysis of placebocontrolled trials for bipolar disorder showed that risperidone, quetiapine, olanzapine, and aripiprazole frequently caused somnolence as an adverse event. ${ }^{29}$ Thus, it is important to keep in mind that even SGAs have a tendency to cause sedation as an adverse event in young patients such as those involved in this study.

In a double-blind comparative study of olanzapine and placebo in UHR patients, McGlashan et al found a significant improvement in those treated with olanzapine from 8 weeks and a faster response to olanzapine compared to placebo. ${ }^{9}$ Similarly, SGAs have been shown to be significantly more effective than placebo in comparative studies in UHR patients from 8 to 12 weeks of treatment, with no difference in safety. ${ }^{9,12,13}$ Thus, the short-term advantage of SGAs in UHR patients is clear. Similar results were found in this study and we suggest that antipsychotics are appropriate for use if immediate improvement is needed. However, the efficacy and safety of SGAs for false positive cases remains uncertain. Therefore, it is important to eliminate adverse events where possible and improve efficacy in the use of SGAs in UHR patients; however, in terms of prescribing SGAs to UHR cases, prescription periods and methods must be studied further.

\section{Conclusion}

Analysis using the GAF, PANSS, and CGI-S showed that UHR patients had higher sensitivity and a faster response to a lower dose of SGAs, compared to patients with FES or MES. We suggest that SGAs can be safely prescribed to UHR patients with extremely mild positive symptoms but with serious acting out, if an appropriate dose is determined and anticholinergic agents are used. These results indicate that it is reasonable to treat UHR patients with SGAs. However, the efficacy and safety of SGAs in these patients was only shown for 12 weeks in this study. Longer-term effects require further study in a larger number of cases and with different evaluation scales.

\section{Disclosure}

The authors report no conflicts of interest in this work.

\section{References}

1. Yung AR, Yuen HP, McGorry PD, et al. Mapping the onset of psychosis: the Comprehensive Assessment of At-Risk Mental States. Aust N ZJ Psychiatry. 2005;39(11-12):964-971.

2. Phillips LJ, Yung AR, McGorry PD, et al. Identification of young people at risk of psychosis: validation of Personal Assessment and Crisis Evaluation Clinic intake criteria. Aust N Z J Psychiatry. 2000; 34(Suppl):S164-S169.

3. Yung AR, McGorry PD, McFarlane CA, Jackson HJ, Patton GC, RakkarA Monitoring and care of young people at incipient risk of psychosis. Schizophr Bull. 1996;22(2):283-303.

4. Marshall M, Lewis S, Lockwood A, Drake R, Jones P, Croudace T. Association between duration of untreated psychosis and outcome in cohorts of first-episode patients: a systematic review. Arch Gen Psychiatry. 2005;62:975-983.

5. Perkins DO, Gu H, Boteva K, Lieberman JA. Relationship between duration of untreated psychosis and outcome in first-episode schizophrenia: a critical review and meta-analysis. Am J Psychiatry. 2005;162(10):1785-1804.

6. McGlashan TH, Walsh BC, Woods SW. The Psychosis-Risk Syndrome. Handbook for Diagnosis and Follow-up. New York, NY: Oxford University Press; 2010.

7. Klosterkötter J, Schultze-Lutter F, Bechdolf A, Ruhrmann S. Prediction and prevention of schizophrenia: what has been achieved and where to go next? World Psychiatry. 2011;10(3):165-174.

8. International Early Psychosis Association Writing Group. International clinical practice guidelines for early psychosis. Br J Psychiatry Suppl. 2005;48:s120-s124.

9. McGlashan TH, Zipursky RB, Perkins D, et al. Randomized, doubleblind trial of olanzapine versus placebo in patients prodromally symptomatic for psychosis. Am J Psychiatry. 2006;163(5):790-799.

10. Walker EF, Cornblatt BA, Addington J, et al. The relation of antipsychotic and antidepressant medication with baseline symptoms and symptom progression: a naturalistic study of the North American Prodrome Longitudinal Sample. Schizophr Res. 2009;115(1):50-57.

11. McGorry PD, Yung AR, Phillips LJ, et al. Randomized controlled trial of interventions designed to reduce the risk of progression to first-episode psychosis in a clinical sample with subthreshold symptoms. Arch Gen Psychiatry. 2002;59(10):921-928.

12. Ruhrmann S, Bechdolf A, Kühn KU, et al; LIPS study group. Acute effects of treatment for prodromal symptoms for people putatively in a late initial prodromal state of psychosis. Br J Psychiatry Suppl. 2007;51: s88-s95

13. Woods SW, Breier A, Zipursky RB, et al. Randomized trial of olanzapine versus placebo in the symptomatic acute treatment of the schizophrenic prodrome. Biol Psychiatry. 2003;54(4):453-464. Erratum in: Biol Psychiatry. 2003;54(4):497.

14. Woods SW, Tully EM, Walsh BC, et al. Aripiprazole in the treatment of the psychosis prodrome: an open-label pilot study. Br J Psychiatry Suppl. 2007;51:s96-s101.

15. Haroun N, Dunn L, Haroun A, Cadenhead KS. Risk and protection in prodromal schizophrenia: ethical implications for clinical practice and future research. Schizophr Bull. 2006;32(1):166-178. 
16. The ICD-10 Classification of Mental and Behavioural Disorders: Diagnostic Criteria for Research. Geneva: World Health Organization; 1993.

17. Kay SR, Fiszbein A, Opler LA. The positive and negative syndrome scale (PANSS) for schizophrenia. Schizophr Bull. 1987;13(2):261-276.

18. National Institute of Mental health . CGI: Clinical global impression. In: Guy W, Bonato RR, editors. Manual for the ECDEU Assessment Battery. 2nd ed. Bethesda, MD: US Department of Health, Education, and Welfare, National Institute of Mental Health; 1970.

19. Endicott J, Spitzer RL, Fleiss JL et al. The global assessment scale. A procedure for measuring overall severity of psychiatric disturbance. Arch Gen Psychiatry. 1976;33(6):766-771.

20. Simpson GM, Angus JW. A rating scale for extrapyramidal side effects. Acta Psychiatr Scand Suppl. 1970;212:11-19.

21. Barnes TR. A rating scale for drug-induced akathisia. Br J Psychiatry. 1989;154:672-676.

22. Munetz MR, Benjamin S. How to examine patients using the Abnormal Involuntary Movement Scale. Hosp Community Psychiatry. 1988;39(11):1172-1177.

23. Lieberman JA, Koreen AR, Chakos M, et al. Factors influencing treatment response and outcome of first-episode schizophrenia: implications for understanding the pathophysiology of schizophrenia. J Clin Psychiatry. 1996;57 Suppl 9:5-9.

24. Buchanan RW, Kreyenbuhl J, Kelly DL, et al; Schizophrenia Patient Outcomes Research Team (PORT). The 2009 schizophrenia PORT psychopharmacological treatment recommendations and summary statements. Schizophr Bull. 2010;36(1):71-93.
25. Chatterjee A, Chakos M, Koreen A, et al. Prevalence and clinical correlates of extrapyramidal signs and spontaneous dyskinesia in never-medicated schizophrenic patients. Am J Psychiatry. 1995;152(12):1724-1729.

26. Lieberman JA, Sheitman B, Chakos M, Robinson D, Schooler N, Keith S. The development of treatment resistance in patients with schizophrenia: a clinical and pathophysiologic perspective. J Clin Psychopharmacol. 1998;18(2 Suppl 1):20S-24S.

27. McEvoy JP, Hogarty GE, Steingard S. Optimal dose of neuroleptic in acute schizophrenia. A controlled study of the neuroleptic threshold and higher haloperidol dose. Arch Gen Psychiatry. 1991;48(8):739-745.

28. Robinson DG, Woerner MG, Alvir JM, et al. Predictors of treatment response from a first episode of schizophrenia or schizoaffective disorder. Am J Psychiatry. 1999;156(4):544-549.

29. Scherk H, Pajonk FG, Leucht S. Second-generation antipsychotic agents in the treatment of acute mania: a systematic review and meta-analysis of randomized controlled trials. Arch Gen Psychiatry. 2007;64(4):442-455. Review.

30. Kane JM, Leucht S, Carpenter D, Docherty JP; Expert Consensus Panel for Optimizing Pharmacologic Treatment of Psychotic Disorders. The expert consensus guideline series. Optimizing pharmacologic treatment of psychotic disorders. Introduction: methods, commentary, and summary. J Clin Psychiatry. 2003;64 Suppl 12:5-19.
Neuropsychiatric Disease and Treatment

\section{Publish your work in this journal}

Neuropsychiatric Disease and Treatment is an international, peerreviewed journal of clinical therapeutics and pharmacology focusing on concise rapid reporting of clinical or pre-clinical studies on a range of neuropsychiatric and neurological disorders. This journal is indexed on PubMed Central, the 'PsycINFO' database and CAS.

\section{Dovepress}

The manuscript management system is completely online and includes a very quick and fair peer-review system, which is all easy to use. Visit http://www.dovepress.com/testimonials.php to read real quotes from published authors. 